\title{
Preface: background and introduction to LEAD
}

Leadership is all around us and is one of the most talked about subjects in business. A search on Amazon.com in April 2015 revealed over 137000 results on leadership alone, and this number has grown exponentially over the last two decades. With so many books already published, what is the need for another leadership book and why write it?

There are many different categories of leadership book ranging from weighty academic tomes and colossal handbooks that are not easily accessible to the practising leader, through to authors who write about their experiences of leadership and the "how to" guides which break complexity down into simple approaches and solutions but lack rigour when examined.

This book attempts to bridge the gap between academia and practice. It provides a real-life narrative encapsulating the development of three business people on a leadership programme whilst explaining the key theories, models and techniques that underpin the leadership methods and approaches deployed. Each of the key chapters is split into two parts. First, the personal stories are written in the style of a novel, allowing ease of access for the reader to associate and empathize with the situations of the real-life leaders. At the end of the dialogue there are a few questions (in italics) for the reader to reflect on to assist them with their own personal leadership development. Secondly, the reader can read the academic underpinning of the leadership learning journey. The reader thus can read the book in a normal linear manner or can read the story without the theory and vice versa.

The primary purpose of our book is to provide a research-based examination of leadership learning in the small-medium sized enterprise (SME) context. Yet the unorthodox approach to this examination is intended to enable readers to stand in the shoes of the owner-manager, director, partner or senior manager (hereafter described as owner/manager). Such readers could be academics, undergraduate, postgraduate and doctoral students. They could be policy-makers and leadership development practitioners. However, we also hope they could be owner/managers: alumni from the 2000 plus participants who want to compare their experiences 
and learning with aspects highlighted in the book; or as a companion for people undertaking the programme; or they could be owner/managers intrigued to explore the topic of leadership and its impact on growth in the small business. It may not be the normal bedtime read ... but for the millions of owner/managers surviving against all odds it could be a useful companion that may just help them to sleep.

\section{BACKGROUND TO LEAD}

Small firms, virtually no matter how they are defined, make up at least 95 per cent of enterprises in the European Community. Within the UK, small-medium sized enterprises (SMEs) account for 99 per cent of all businesses (Carter and Jones-Evans, 2006). Today even the casual newspaper reader knows about the key role which small firms play in employment creation, their overall importance in the economy, their role in innovation and the importance which government attaches to "enterprise" (Storey, 1994, p. 1). By just about any measure the contribution small firms make to the economy of any country is increasing and their importance is now fully recognized (Burns, 2007). A flourishing small business sector is central to economic growth.

The leadership of the owner/manager is seen to have an impact on the performance of the business. The management and leadership capability is thus a key factor in SME survival and growth.

The LEAD programme (hereafter referred to as LEAD) was developed by the Institute for Entrepreneurship and Enterprise Development within Lancaster University Management School in 2004. At this time, policy was recognizing and pushing for the need for universities to support SMEs and develop their leadership. One of the government's key aims as outlined in the Skills White Paper is to improve leadership and management capability: "Effective leadership and management are key to the development of competitive businesses" (DfES, 2003, chapter 2, 2.14).

The Framework for Regional Employment and Skills Action identified the need to take action to address regional management capability, not only because it affects performance and productivity of individual companies, but also because it impacts on the ability of business leaders to address the skills gap by managing wider skills development within these businesses (Framework for Regional Employment and Skills Action, 2002).

LEAD was designed to benefit the region's micro companies by providing access to the highest quality leadership development to 
individual owner/managers. ${ }^{2}$ The SME sector is under-represented in training programmes with most, if not all, other training programmes being designed for and marketed to managers within larger companies (Smith and Peters, 2006). The main objective of LEAD (a key objective still today) was to raise regional productivity, competitiveness and skills by addressing issues of leadership within the context of the SME sector generally and in particular within the owner/manager's business.

Although initially born out of Lancaster University, LEAD has over the past ten years been delivered across the United Kingdom by other Higher Education Institutions, Further Education Colleges and private training companies to over 2000 SMEs. Steve has provided guidance to the Australian Government linked with the Australian Family Business Centre at Bond University to design a similar programme. Finally the programme is being designed for delivery in partnership with Porto Business School in the spring of 2015 with discussion on-going with many other partners.

Whilst leadership is all around us, it is a subject that arguably means little to the person or persons running a small business even though they are "doing" leadership each and every day.

The challenge for the small business leader is whether to work "in" the business, providing day-to-day management support or "on" the business, providing strategic leadership, to enable growth. Research shows most do the former, finding it difficult to remove themselves from the operational concerns to focus on longer-term strategy (Jones et al., 2007).

LEAD is a research-led programme, based on extensive research into entrepreneurial leadership learning in SMEs (Kempster and Watts, 2002; Cope, 2003). The major contribution of this research was the identification that owner/managers are structurally disadvantaged in their leadership learning (Kempster, 2005; Smith and Peters, 2006; Kempster, 2009a; Smith, 2011) as a consequence of the small business context and the antecedent lived experience of the owner/managers such as feelings of isolation, limited confidence, distant relationship with leadership, prominent patriarchal style, control and limited role models (Perren and Grant, 2001; Smith et al. 1999). Stewart and Alexander (2006, p. 143) argue that the isolation of the enterprise owner/manager is a barrier to learning because they may be the only person within the company concerned with strategic decision-making, yet it is precisely the competencies of the owner/manager that directly impact on the survival and success of an enterprise.

Burgoyne and Stewart (1977) identified the notion of naturalistic

${ }^{2}$ Lancaster University Management School is one of two six star rated management schools in the UK. 
learning as formative in shaping leadership learning and that leadership is primarily learnt through experience (Kempster, 2006) by actually trying to lead (Jackson and Parry, 2011). The design of LEAD responds to this.

The fundamental assumption made by LEAD is that any process for the leadership development of the owner/manager must be integrated with the business. This is because the senior management in SMEs typically are the business and the business reflects the senior management. Leadership is learnt within organizational contexts and leaders learn through the everyday activities they participate in. Yet because it is everyday life, leaders often cannot put their finger on how they have become the leaders that they are.

There is often a major disconnect between "what someone thinks leadership is" - their intent - and "how a person learns to practise leadership" their impact. LEAD seeks to bridge this disconnect by connecting the awareness of leadership and learning with leadership practice, that is, how a leader leads within their business.

LEAD was designed on the premise that business growth and business development would occur if leadership became more salient to the owner/ manager and the sense of leadership identity of owner/managers would become enriched, nuanced and relevant in the context of their own business and own lives (Kempster, 2005).

LEAD combines formal input and experiential learning within a peer group of other SME leaders. A "LEADership Learning Cycle through Lived Experience" is proposed (Figure P.1) integrating a combination of formal and situational learning to develop the owner/manager and the business through a variety of management and leadership development activities. This integrated process of "lived experience" over a ten-month period has been found to be critical to the success of LEAD as business people in SMEs do not typically identify themselves as a "leader" (Kempster and Watts, 2002).

From the outset, LEAD explicitly makes leadership highly salient to delegates in their organizational setting, building on the notion of learning about leadership as a deepening cycle of experience as the delegates shape and are shaped by the situation. It is also essential that the owner/manager be given the tools and transferable skills, for example coaching, to be able to develop and instil leadership within their teams, shaping both their own learning and that of others in the organization.

The cyclical model commences, if a starting point could be so easily identified, with reflection-on-action (Schön, 1983) which makes sense of an experience from which knowledge conceptualization follows. Experiences of leadership generate knowledge and understanding of leadership. Such 


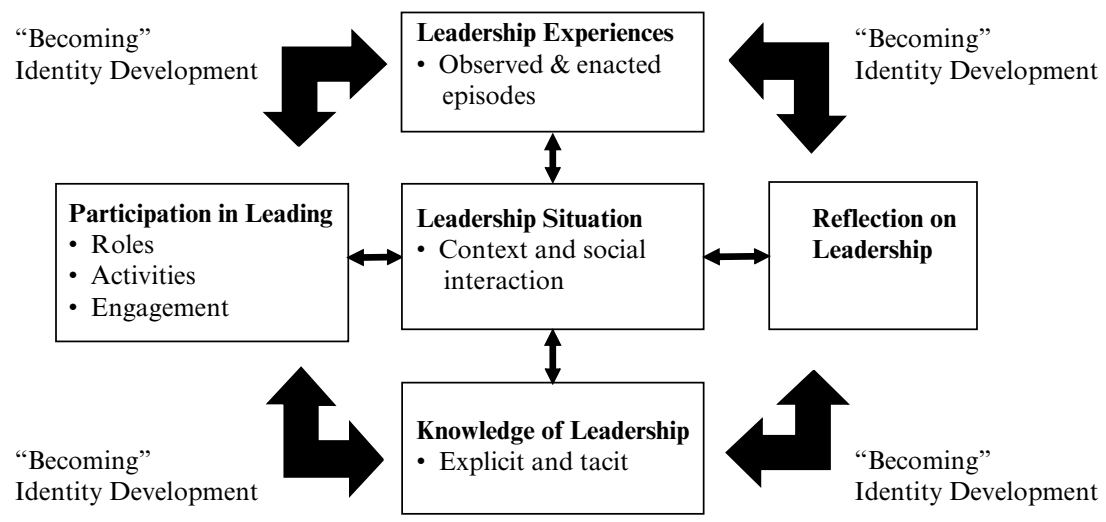

Source: Adapted from Kempster (2009a). Used by permission of the publishers, Palgrave Macmillan.

\section{Figure P.1 LEADership Learning Cycle through lived experience}

understanding relates to the situation from which it is drawn. This stimulates reflection from which knowledge is constructed. This knowledge, in the form of a personalized understanding of leadership, is subsequently applied and generates further experiences through participation that through reflection refine, elaborate or confirm knowledge of the experience. There is a move from understanding leadership to participating in leading and how lived experience is central to leadership learning.

This book seeks to outline why LEAD has been successful, establishes the context for small business leadership development, and describes the key dynamics of leadership learning and why it is different in the SME context.

Throughout, we explore the learning journey of three SME business leaders through a series of in-depth, semi-structured interviews aided by formal outputs of LEAD produced by the interviewees during and after the programme, and we draw on their reflective online forum blogs (an integral part of the programme design).

The approach to this research draws on a research method called "coconstructed autoethnography" (Ellis and Bochner, 2000; Boyle and Parry, 2007). Although it is not a common method in management research, Kempster and Stewart (2010) have argued that it is most relevant to the study of leadership learning as it allows the connection of context, historic antecedent influences and relationships to be examined in-depth to both describe and explain the manifestations of particular social processes (Atkinson et al., 2003). Such detailed qualitative research is argued to be 
a highly appropriate approach for explorations of leadership processes (Bryman, 1996, 2004; Parry, 1998; Day, 2000; Conger, 2004; Kempster, 2006).

It may seem odd that somehow good research can be achieved by examining case study stories of three owner/managers. However, Van Maanen wonderfully captures the essence of the approach: "the universal can be found in the particular" (2011, p. 229). Further, Boyle and Parry suggest that research needs to give more emphasis to relevance and connectivity of ideas and insights that resonate with readers' lives. "We would suggest that the critical ' $n$ ' factor is the number of people who read the research, rather than the number of people who are the subjects of the research" (2007, p. 6).

Similarly Ellis and Bochner (2000) suggest that generalization is tested by readers as they determine if it speaks to them about their experience or about the lives of others they know. Stake (2005) interprets such generalization as naturalistic: "a sense of empathetic appreciation from one world to another that provides a vicarious experience for the reader" (Kempster and Stewart, 2010, p.210). With respect to the discussion in this book, we are seeking to provide a sense of an appearance of authenticity through rich insights into leadership learning of three owner/managers coping with the challenges of leader becoming (Ibarra, 1999).

When we speak of becoming, we are giving emphasis to notions of a relational sense of the self that is emerging through participation. The participation with others shapes us and others so that our becoming is inextricably linked to others in terms of being, knowing and doing (Shotter, 1993; Chia and MacKay, 2007). In this way we depart from an attention to becoming associated with essentialist qualities of an individual - such as traits. For example, the knowing, doing and being associated with becoming a leader is informed by historic practices and constructed through what others do, say and respond to; it is an on-going dynamic of becoming rather than a static sense of being (Alvesson and Sveningsson, 2003; Sveningsson and Larsson, 2006).

In brief, autoethnography examines the ethno, the three owner/managers in the context of being a delegate on LEAD and situated in their businesses. While the graphy describes the social engagement on LEAD integrated with the on-going leadership of their businesses for a period of ten months from March to December. If it was each of the owner/ managers examining their experiences and distilling social theory this would be autoethnography - their accounts. Co-constructed is where we examine, interrogate and critique in depth what has occurred in greater depth. We bring the theory to be applied to what is happening to extract the social processes occurring. In this way the theory becomes relevant in 
the examination. Ellis describes this process as a "theory sandwich" (2004, p. 198) - a sense of a continual re-appraisal and interpretation of the story through theorizing (Kempster and Stewart, 2010, p. 210).

The layout of the book reflects this theory sandwich. We first provide the story from the three cases of Freddie, Jane and Bill. Then we subsequently examine the stories using selected and relevant theory.

Data was collected through access to over 150 pages of data regarding Freddie, Jane and Bill. This was from: online LEAD Forum notes, learning logs, contemporaneous notes from coaching and action learning, outputs from the Shadowing/Exchange and Learning Days. Additionally we examined their own meta-reflections contained in their 8000-word submissions of their Work Based Learning when they had their Master's level learning assessed. Using the findings from the data as a guide, semistructured interviews were held with the three owner/managers in their offices, lasting twelve hours in total, where they each reflected on what they experienced, how they felt, what changes they were making in their business at each stage and what changes in themselves they were beginning to experience. The interviews were recorded and transcribed into 92 pages containing over 62000 words which were compared to the other data collected and used in the narrative in each chapter to describe their unfolding stories. Notably the vocabulary of the three interviewees changed during LEAD as they adopted and applied some of the language learned in their everyday conversations. For example, the delegates spoke to their staff about "open questions" from "Action Learning Sets" and imagining a "Future Perfect" state which is from Coaching.

Our research approach to examining this data sought a combination of both evocative and analytic autoethnography. Evocative autoethnographic research seeks to "[move] the reader to feel the feelings of the other" (Denzin, 1997, p. 228). We see much value in enabling a reader to stand in the shoes of the interviewees to gain a deep insight into the experience, a sense of believability. Analytic autoethnography (Anderson, 2006, p. 376) seeks to go beyond the individual's experience and look for social processes and influences that are common in shaping leadership learning more generally. We hope the approach of the theory sandwich as research enables a deep sense of standing in the shoes of these three owner/ managers and also gives a sense of the theory that explains the social processes occurring. We have sought to keep the theory to a minimum so that the sandwich does not end up feeling like having a dried out tough bit of meat ruining the sandwich. We hope we have struck the right balance.

LEAD has made a hugely positive impact on the business for the vast majority of participants. Three large-scale evaluations of LEAD have been conducted looking in particular at the personal, business and economic 
impact of the programme (Wren and Jones, 2006, 2012; Gill and Harris, 2014). The assessments identified an overwhelmingly positive set of statistics. Among these, significant increases in annual sales, employment and profitability or profits were reported by 97 per cent of participating firms with an average increase in turnover of 27 per cent and an increase in employment of 13 per cent. Delegates also reported increased confidence in and awareness of: individuals' leadership roles; elevated abilities to delegate effectively, leading to staff empowerment; and a wider approach to innovation as evidenced by expansion, relocation, diversification and acquisition activities.

The result of all this has been to free up time for owner/managers to work more on their businesses, and less in their businesses.

We conclude the story of LEAD with the policy implications of the programme in terms of economic growth through the dynamo of an economy - small business and the owner/manager. If policy-makers are committed to the growth of SMEs, then we argue that they need to make a commitment to developing the leadership of SMEs through the promotion and part funding of programmes such as LEAD. Economic payback in such short periods demonstrably drives growth and employment.

Finally, in an epilogue, we share how the business leader can continue their development journey on the subsequent GOLD and GAIN programmes and we also discuss how the owner/manager, through Work Based Learning, can progressively achieve a Postgraduate Certificate, Diploma and Master's degree by having their learning recognized to essentially obtain a Master's in running their own company.

\section{INTRODUCTION TO LEAD}

At the beginning of LEAD it's all a bit strange and different for delegates. LEAD is not a conventional taught "training" course; it is about the development of the leader and the development of their business. Delegates are encouraged to "trust the process" and to give themselves time to reflect, time to learn and time to enjoy the programme.

LEAD lasts for approximately ten months and delegates need to commit an average of two days a month (or part thereof).

LEAD employs the LEADership Learning Cycle through Lived Experience integrating a number of key elements that combine both formal (that is, taught) and informal learning, allowing for a high level of interaction between delegates and the LEAD team and, crucially, between delegates and their fellow cohort members. The key elements are: 
1. Overnight Experiential

2. Masterclasses

3. Action Learning Sets

4. Coaching

5. Business Shadowing and Exchanges

6. Online LEAD Forum

7. Learning and Reflection Days

The programme commences with an Induction and concludes with a Graduation.

LEAD is rooted in a participative teaching methodology. It supports a social view of learning that relies upon peer-to-peer learning to make sense of the different learning interventions. This pedagogy is based on constructionist views of knowledge which requires the delegates to engage with the ideas that come from the different elements of LEAD and to develop skills and capabilities relevant to their own situations back in their businesses. This pedagogy includes learner-directed styles of learning and interactive approaches for the delegates to learn from each other and the knowledge they have about running small businesses.

LEAD uses a range of delivery approaches:

- Leadership situation - the context of a real, lived and situated experience is placed centrally as both a catalyst of available learning opportunities and as a filter shaping a delegate's learning. In essence, this is where the delegate "does" leadership on a daily basis.

- Knowledge of leadership - understanding of leadership becomes interrelated with the situation and subsequently allows the delegate to interpret the situation to provide guidance or prompts to leadership approaches.

- Participation in leading - engagement with leadership is within situations. Participation through roles and associated activities creates opportunities for experiences of leading and, through participation, knowledge of detailed nuances of leading in a particular situation may be absorbed both explicitly and tacitly.

- Leadership experiences - the variety and difference of experiences of leading drawn from situations enable greater development to occur through learning from observation and enacting leadership.

- Reflections on leadership - the overt role of reflection as a continual conscious process enables the delegate to surface and make sense of their activities which develops their individual leadership. 


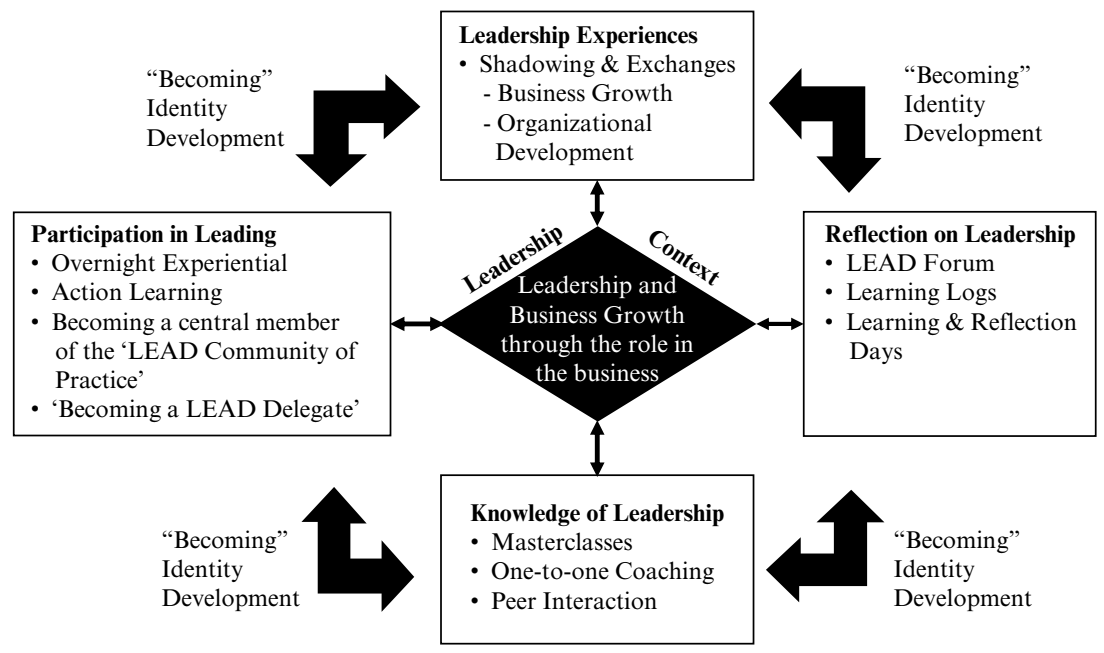

Figure P.2 Structure of the LEAD programme - "becoming" a leader

Each of the above five dimensions of the LEADership Learning Cycle is the basis of at least one of the elements of LEAD (see Figure P.2).

This is the central model that runs through the book - like a red thread - connecting the theoretical understanding together alongside the examination of leader becoming of the three owner/managers. For us it is much more than a set of individual aspects. We outline in the Chapter 10 theory sandwich the metaphors of marbles and a bird's nest. We would recommend jumping ahead to this theory sandwich to get an understanding of the metaphor. It speaks to the importance of a holistic appreciation of how the learning is integrated and reinforces each aspect rather than stand-alone elements.

The delegates' everyday social setting, their workplace, is situated at the centre of the LEADership Learning Cycle - Lived Experience. This leadership situation is the crucible of leadership (Bennis and Thomas, 2002) where their identity as a leader has been formed and where it unconsciously continues to develop in the everyday happenings of business. On LEAD, delegates are encouraged to consider how the range of delivery approaches - Participation in Leading, Knowledge of Leadership, Leadership Experiences and Reflection on Leadership - impact on them and the Leadership Situation in the context of their business.

There is a strong emphasis on reflective learning over the duration of the programme. LEAD encourages delegates to implement new ideas in their businesses and bring their experiences of this back to share with their 
cohort. Thus, delegates bring to the learning table both their experience of practice and their experience of themselves in that practice.

Throughout LEAD, delegates are asked to keep a learning log, which is a private and confidential record for them to plan and document their own personal development and the changes they implement in their business while they are on LEAD. It gives delegates the opportunity to record, review and plan what they do during their time on LEAD; it demonstrates examples of how delegates are putting skills into practice; it provides an insight into the delegates' preferred ways of learning and increasing subject knowledge; it encourages a greater sense of confidence, self-awareness and identity; it acts as a benchmark throughout LEAD on where delegates are in achieving their learning objectives. 